\title{
Prenatal and postnatal exposure to acetaminophen in relation to autism spectrum and attention-deficit and hyperactivity symptoms in childhood: Meta-analysis in six European population-based cohorts
}

\author{
Silvia Alemany 1,2,3 (Claudia Avella-García ${ }^{1,2,3,4} \cdot$ Zeyan Liew $^{5,6} \cdot$ Raquel García-Esteban $^{1,2,3} \cdot$ Kosuke Inoue $^{7}$. \\ Tim Cadman ${ }^{8,9} \cdot$ Mònica López-Vicente $^{10}$ • Llúcia González ${ }^{3,11}$ • Isolina Riaño Galán ${ }^{3,12}$. Ainara Andiarena ${ }^{13,14}$. \\ Maribel Casas ${ }^{1,2,3} \cdot$ Katerina Margetaki ${ }^{15}$. Katrine Strandberg-Larsen ${ }^{16}$ • Deborah A. Lawlor ${ }^{8,9,17}$. \\ Hanan El Marroun ${ }^{10,18,19}$. Henning Tiemeier ${ }^{10,20}$. Carmen Iñiguez ${ }^{21,3,11}$. Adonina Tardón ${ }^{3,22}$. \\ Loreto Santa-Marina ${ }^{3,23,24} \cdot$ Jordi Júlvez ${ }^{1,2,3,25} \cdot$ Daniela Porta $^{26} \cdot$ Leda Chatzi $^{27} \cdot$ Jordi Sunyer ${ }^{1,2,3,28}$
}

Received: 19 October 2020 / Accepted: 20 April 2021 / Published online: 28 May 2021

(c) The Author(s) 2021

\begin{abstract}
The potential etiological role of early acetaminophen exposure on Autism Spectrum Conditions (ASC) and Attention-Deficit/ Hyperactivity Disorder (ADHD) is inconclusive. We aimed to study this association in a collaborative study of six European population-based birth/child cohorts. A total of 73,881 mother-child pairs were included in the study. Prenatal and postnatal (up to 18 months) acetaminophen exposure was assessed through maternal questionnaires or interviews. ASC and ADHD symptoms were assessed at 4-12 years of age using validated instruments. Children were classified as having borderline/ clinical symptoms using recommended cutoffs for each instrument. Hospital diagnoses were also available in one cohort. Analyses were adjusted for child and maternal characteristics along with indications for acetaminophen use. Adjusted cohortspecific effect estimates were combined using random-effects meta-analysis. The proportion of children having borderline/ clinical symptoms ranged between 0.9 and $12.9 \%$ for ASC and between 1.2 and 12.2\% for ADHD. Results indicated that children prenatally exposed to acetaminophen were $19 \%$ and $21 \%$ more likely to subsequently have borderline or clinical ASC $(\mathrm{OR}=1.19,95 \%$ CI 1.07-1.33) and ADHD symptoms ( $\mathrm{OR}=1.21,95 \%$ CI 1.07-1.36) compared to non-exposed children. Boys and girls showed higher odds for ASC and ADHD symptoms after prenatal exposure, though these associations were slightly stronger among boys. Postnatal exposure to acetaminophen was not associated with ASC or ADHD symptoms. These results replicate previous work and support providing clear information to pregnant women and their partners about potential long-term risks of acetaminophen use.
\end{abstract}

Keywords Acetaminophen $\cdot$ Paracetamol $\cdot$ Pregnancy $\cdot$ Autism $\cdot$ Attention-deficit/hyperactivity disorder

\section{Introduction}

Acetaminophen (or paracetamol) is used by $46-56 \%$ of pregnant women in developed countries [1-3]. Whilst acetaminophen is considered the safest analgesic/antipyretic for pregnant women and children, mounting evidence has linked prenatal acetaminophen exposure to worse cognitive performance [4-6], more behavioural problems [1, 7, 8], Autism Spectrum Conditions (ASC) $[6,9]$ and Attention-Deficit/

Silvia Alemany

silvia.alemany1983@gmail.com

Extended author information available on the last page of the article
Hyperactivity Disorder (ADHD) symptoms [1, 3, 6, 10]. Recently, detection of acetaminophen in meconium has associated with increased odds of ADHD and altered frontoparietal connectivity at ages 9-10 years [11].

Two meta-analyses have investigated the link between prenatal acetaminophen use and ASC and ADHD symptoms $[12,13]$. The first meta-analysis included seven cohort studies $(\mathrm{N}=132,738)$ and reported risk increases of $19 \%$ for ASC and 34\% for ADHD [12]. The second metaanalysis focused on ADHD, included eight cohort studies $(\mathrm{N}=244,940)$ and concluded that exposed children had a $25 \%$ increased risk of developing ADHD symptoms [13]. However, the methods and instruments used to assess the outcome in the studies included in the meta-analysis are 
highly heterogeneous $[14,15]$. Another source of heterogeneity among these studies regards the statistical approach and confounders included [14]. Furthermore, these studies did not address relevant unsolved questions regarding the link between early acetaminophen exposure and ASC and ADHD symptoms.

First, it is unclear whether girls or boys are differentially affected by acetaminophen exposure. A recent study examining biomarkers of prenatal acetaminophen exposure among 996 mother-child pairs observed higher odds for ASC among boys and higher odds for ADHD among girls. [16] This is partially in agreement with a population-based study conducted in INfancia y Medio Ambiente (INMA) cohort representing 2644 mother-child pairs, where prenatal acetaminophen exposure was positively associated with ASC symptoms only among boys. [6] However, two studies including 64,322 mothers enrolled in the Danish National Birth Cohort (DNBC), reported slightly higher estimates among girls for ADHD and ASC without hyperkinetic symptoms [1, 9]. Second, the abovementioned studies on INMA cohort [6] and acetaminophen biomarkers [16] observed positive associations with both ASC and ADHD symptoms. This contrasts with the study conducted in the DNBC cohort where prenatal exposure was linked to ASC when accompanied by hyperkinetic symptoms [9]. Finally, another gap in research relates to postnatal exposure. An ecological study found positive correlations between indicators of both prenatal and postnatal acetaminophen exposures and ASC prevalence [17]. To our knowledge, postnatal associations have not been examined in prospective cohort studies.

In the current study, we aim to examine the association between early acetaminophen exposure and ADHD and ASC symptoms and hospital diagnosis. Furthermore, we also examined postnatal exposure and we present sex-stratified results. To reduce heterogeneity, we used a common set of confounders, the same statistical approach and harmonized the exposure and outcomes measurements across cohorts.

\section{Methods}

\section{Sample}

We included six European population-based birth cohorts: Avon Longitudinal Study of Parents and Children (ALSPAC), DNBC, Gene and Environment: Prospective Study on Infancy in Italy (GASPII), the Generation R Study, INMA (including four subcohorts), and the Mother-Child Cohort in Crete (RHEA) (Methods S1). Mother-child pairs were recruited from 1991 through 2008. A total of 73,881 children with available data on either prenatal or postnatal exposure to acetaminophen and at least one outcome (ASC or ADHD symptoms) and main covariates were included ( $60.5 \%$ of the children recruited at baseline).

Informed consent was obtained from all participants in each cohort and ethical approval was obtained from the local authorized institutional review boards.

\section{Exposure}

Regarding prenatal acetaminophen exposure, mothers were interviewed two (INMA, RHEA), three (GASPII) or four (DNBC) times during pregnancy using standardized questionnaires. At each interview, mothers were asked if they had taken medications from the month before becoming pregnant (GASPII; INMA) or beginning of pregnancy (RHEA) through delivery. In ALSPAC and the Generation R Study, mothers completed questionnaires two (ALSPAC) or three (the Generation R Study) times during pregnancy reporting acetaminophen use from the month before becoming pregnant to gestational week 32 of pregnancy. In GASPII, mothers were interviewed at birth and provided retrospective information on acetaminophen use at each trimester. Mothers were classified as ever exposed if they reported having taken any dose of acetaminophen in the defined prenatal exposure period; otherwise they were classified as nonexposed (More details in Table 1 and Supplemental Materials (S) Methods S2).

Regarding postnatal acetaminophen exposure, mothers were interviewed (DNBC, GASP, RHEA) or completed questionnaires (INMA) about medication use in their children one (RHEA) or two times (The Generation R Study, GASP, INMA, DNBC) in the first 18 months of life of the child. At each interview, mothers were asked if they had given any medication including acetaminophen, to their child. Children were classified as postnatally exposed to acetaminophen if they had taken any dose of acetaminophen at any time up to 18 months of life. Otherwise, they were considered non-exposed (Table 1, Methods S3).

\section{Outcomes}

ASC and ADHD symptoms were assessed using validated parent-reported questionnaires or linked hospital records. Autistic symptoms were assessed using the Development And Well-Being Assessment (DAWBA) [18] (ALSPAC), the Pervasive Developmental Problems (PDP) subscale of the Child Behaviour Checklist for Toddlers (CBCL1 $1 \frac{12-5)}{2}$ [19] (GASPII and The Generation R Study), the Childhood Autism Spectrum Test (CAST) [20] (INMA) and an ASC scale derived from the CBCL for 6-18 (CBCL6-18) [21] (RHEA). ADHD symptoms were assessed using the Development and Well-Being Assessment (DAWBA) [18] (ALSPAC); the Conner's Parent Rating Scale Revised short form (CPRS-R:S) [22] (The Generation R Study), 
Table 1 Assessment of exposures

\begin{tabular}{|c|c|c|c|c|c|c|c|c|}
\hline \multirow[t]{2}{*}{ Cohort study } & \multirow[t]{2}{*}{ Pregnancy period } & \multirow[t]{2}{*}{$\mathrm{N}^{\mathrm{a}}$} & \multicolumn{3}{|l|}{ Prenatal } & \multicolumn{3}{|l|}{ Postnatal } \\
\hline & & & Instrument & N Exposed & $\%$ Exposed & Instrument & N Exposed & $\%$ Exposed \\
\hline ALSPAC & 1991-1992 & 6200 & $\begin{array}{l}\text { Maternal questionni- } \\
\text { are completed at } \\
18 \text { and } 32 \text { weeks of } \\
\text { pregnancy }\end{array}$ & 2425 & 39.1 & $\begin{array}{l}\text { Maternal questionniare } \\
\text { completed when chil- } \\
\text { dren were } 12 \text { months } \\
\text { old }\end{array}$ & 375 & 6.0 \\
\hline DNBC & 1996-2002 & 61,430 & $\begin{array}{l}\text { Self-reported study } \\
\text { enrolment form and } \\
\text { in three computer- } \\
\text { assisted telephone } \\
\text { interviews (12th } \\
\text { and 30th week, and } \\
\text { 6-month postpartum) }\end{array}$ & 34,584 & 56.3 & $\begin{array}{l}\text { Computer-assisted } \\
\text { telephone inter- } \\
\text { views with moth- } \\
\text { ers when children } \\
\text { were } 6 \text { months and } \\
18 \text { months old }\end{array}$ & 4735 & 7.7 \\
\hline GASPII & 2003-2004 & 489 & $\begin{array}{l}\text { Interview with mothers } \\
\text { at first, second and } \\
\text { third trimesters of } \\
\text { pregnancy }\end{array}$ & 153 & 31.3 & $\begin{array}{l}\text { Interview with moths } \\
\text { when children were } 6 \\
\text { and } 15 \text { months old }\end{array}$ & 454 & 92.8 \\
\hline Generation $\mathrm{R}$ & 2001-2005 & 3904 & $\begin{array}{l}\text { Maternal questionniare } \\
\text { completed at } 12, \\
20 \text { and } 30 \text { weeks of } \\
\text { pregnancy }\end{array}$ & 1150 & 29.5 & $\begin{array}{l}\text { Maternal questionniare } \\
\text { completed when chil- } \\
\text { dren were } 12 \text { months } \\
\text { old }\end{array}$ & 2526 & 64.7 \\
\hline INMA & 2004-2008 & 1513 & $\begin{array}{l}\text { Interview with mothers } \\
\text { at weeks } 12 \text { and } 32 \text { of } \\
\text { pregnancy }\end{array}$ & 775 & 51.2 & $\begin{array}{l}\text { Maternal ques- } \\
\text { tionnaires when } \\
\text { children were } 6 \text {, } \\
14 \text { or } 18 \text { months } \\
\text { old (depending on } \\
\text { subcohort) }\end{array}$ & $750^{\mathrm{b}}$ & $90.8^{b}$ \\
\hline RHEA & 2007-2008 & 345 & $\begin{array}{l}\text { Interview with mothers } \\
\text { at weeks } 12 \text { and } 30 \text { of } \\
\text { pregnancy }\end{array}$ & 49 & 14.2 & $\begin{array}{l}\text { Interview with mothers } \\
\text { when children were } \\
9 \text { months old }\end{array}$ & 306 & 88.7 \\
\hline
\end{tabular}

${ }^{a}$ Number of children with data available on early acetaminophen exposure (prenatal or postnatal), at least one outcome (ASC or ADHD symptoms) and main covariates

${ }^{b}$ Number of children with data available for postnatal exposure, at lest one outcome and main covariates is 829

the Hyperactivity/Inattention subscale of the Strengths and Difficulties Questionnaire (SDQ) [23] (DNBC), the Attention Deficit and Hyperactivity problems subscale of the CBCL1 1 $12-5$ and CBCL6/18 (GASPII and RHEA), and the ADHD Criteria of DSM-IV (DSM-ADHD Questionnaire) [24] (INMA). Higher scores indicate more symptoms.

In addition to the SDQ-Hyperactivity/Inattention subscale, in the DNBC cohort, diagnoses of ASC and ADHD from national hospital register were also available [1].

To harmonize the continuous scores on ASC and ADHD symptoms, we used questionnaire-specific validated cutoffs to yield proxies for ASC and ADHD symptoms within the borderline/clinical range. Specifically, for the DSMADHD Questionnaire scores, we applied established cut-offs consisting of 6 or more symptoms of inattention or hyperactivity to yield proxies for borderline/clinical ADHD symptoms [24]. For CBCL1 1 $12-5$ and CBCL6/18 scales, we used the recommended cut-off to classify children with borderline/clinical symptoms corresponding to scores above 93 rd percentile $[25,26]$. Of note, since no specific cut-offs have been established for the ASC-CBCL scale [21], we applied the recommended cut-off abovementioned for the CBCL6/18 scales. ${ }^{25}$ The ADHD index of the CSRS-R:S has a cut-off for elevated T-scores (65-69) that we used to classify children as presenting borderline/clinical symptoms (T-scores $>65$ ) [22]. The DAWBA is based on diagnostic criteria (ICD-10 and DSM-IV) and focuses on anxiety disorders, depressive disorders, ADHD and conduct disorders. A clinical diagnostic rating is informed by triangulation of these three sources [27]. In the case of SDQ, abnormal scores in emotional problems and in peer problems or prosocial behaviour subscales were used to capture ASC borderline/clinical symptoms and abnormal scores in the SDQ hyperactivity/inattention and impact subscales were used to capture ADHD borderline/clinical symptoms [28]. Finally, for SCDC scores, the cut-off of 8 to detect probable cases was applied to classify children as having borderline/clinical ASC symptoms [29]. Further details on outcomes measures and cut-offs applied can be found in Table 2 and Methods S4-S5. A summary of the 


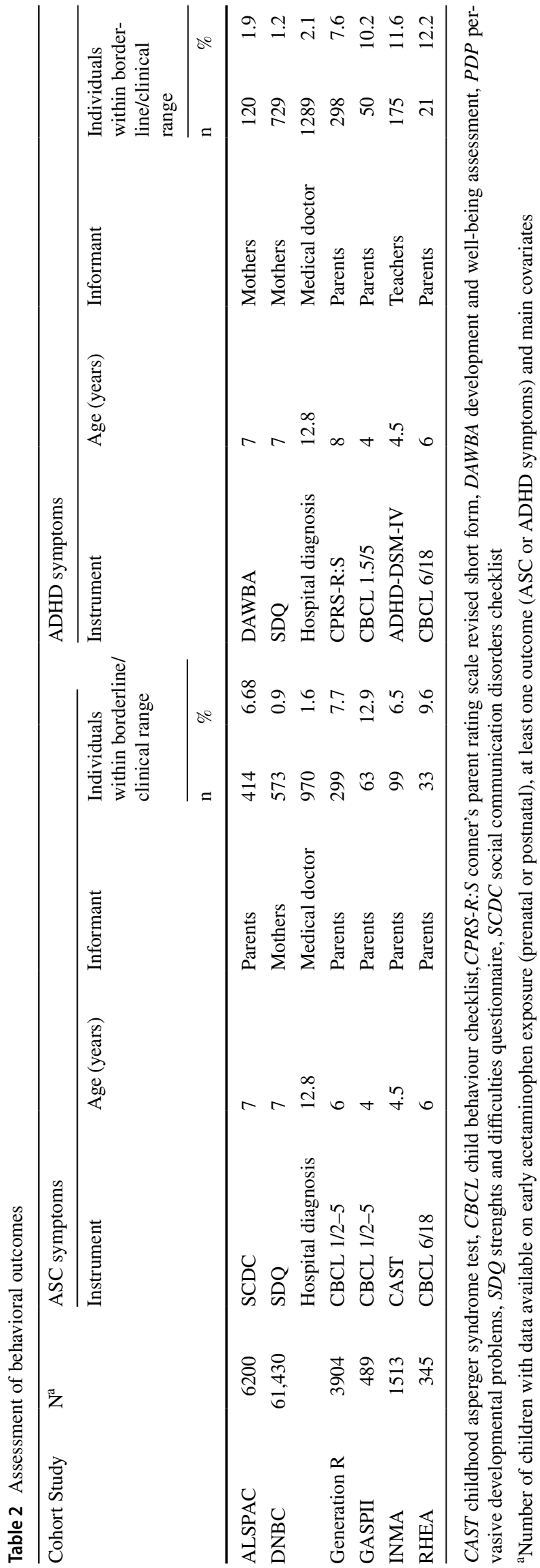

psychometric properties of the instruments used to assess the outcomes can be found in Table S1.

\section{Covariates}

Potential confounding variables were selected a priori prioritizing availability and consistency. Covariates were factors previously associated with ASC, ADHD and acetaminophen exposure, and include maternal and child characteristics. Maternal characteristics included age at delivery (years), education (low, medium, high), pre-pregnancy body-mass index (BMI), alcohol (yes/no), smoking (yes/no) and mental health problems (yes/no) during pregnancy, age at birth (years) and parity (nulliparous, $>1$ and $>2$ ), maternal fever (yes/no) and infections (yes/no) during pregnancy. Maternal education was not provided by the DNBC and analyses were adjusted by maternal socio-occupational status based on job titles instead. Child characteristics included sex, age at behavioural assessment (years), cold (yes/no) and respiratory infections (yes/no) in the first 2 years of life. Maternal characteristics were collected during pregnancy except for mental health in INMA, which was collected when children were around 5 years. Child characteristics were collected in the first 18 months of life. Two cohorts adjusted their analysis for cohort-specific covariates. Specifically, analysis in Generation R Study were also adjusted by ethnicity (Dutch, non-Dutch/other western, non-western) and analysis in INMA were also adjusted by subcohort (INMA-Asturias, INMA-Gipuzkoa, INMA-Sabadell and INMA-Valencia).

\section{Statistical analysis}

All analyses were performed following the same protocol. We used logistic regression models to assess the association between prenatal and postnatal acetaminophen exposure and ASC or ADHD symptoms (as binary outcomes) within the borderline/clinical range. Separate models were conducted for prenatal and postnatal exposure, and for ASC and ADHD symptoms. Main models were adjusted for the abovementioned covariates and stratified by child sex.

Cohort-specific analyses were undertaken at each study centre and pooled using random-effects meta-analysis. Random effects models, which account for unexplained heterogeneity, assume that the effect size varies across the studies because of real differences in the exposure effect and sampling variability [30]. Between-study heterogeneity was assessed using Cochran's $Q$ test and the $I^{2}$ statistic.

Since the cohorts included herein differ in the length of follow-up, we performed meta-regression including mean age of the child at outcome assessment as moderator. These analyses allow us to examine potential changes in the association between acetaminophen exposure and ASC and ADHD symptoms over time. 
Sensitivity analyses included: (a) testing the associations with hospital diagnosis of ASC and ADHD available in the DNBC cohort, (b) testing the associations with ASC symptoms excluding ADHD cases, (c) meta-analyses leaving out one cohort at a time to determine the influence of each cohort, and (d) additional adjustment for gestational age, birthweight (grams), maternal chronic diseases -except psychiatric diseases- (yes/no), maternal use of other drugs (yes/no) and maternal folic acid use (yes/no).

Analyses were performed using R 3.5.1 (https://www.rproject.org/). Logistic regression models to estimate odds ratio (OR) and 95\% confidence interval (CI) were fitted using finalfit package (https://finalfit.org/index.html). Metaanalyses were conducted using metafor package [31].

\section{Results}

Across studies between 14 and $56 \%$ of mothers reported acetaminophen use in the prenatal period, with the RHEA cohort having the lowest proportion and DNBC the highest. A wider range of child postnatal acetaminophen exposure was reported varying from 6\% in ALSPAC to $92.8 \%$ in GASPII (Table 1).

The total number of children having ASC or ADHD symptoms within the borderline/clinical range was 1,481 $(2.1 \%)$ and $1,393(2 \%)$, respectively (Table 2 ). Across cohorts, we observed large variations in the proportion of children presenting borderline/clinical symptoms with $0.9 \%$ and $12.9 \%$ presenting ASC symptoms and between 1.2 and $12.2 \%$ presenting ADHD symptoms.

Table 3 shows the distributions of maternal and child characteristics.

Children with ASC symptoms within the borderline/clinical range were more likely to be males and their mothers were more likely to be younger, have lower educational level and report alcohol consumption and mental health problems during pregnancy compared to children not in the borderline/ clinical range (Table S2). Children with ADHD symptoms within the borderline/clinical range were also more likely to be males and have a higher proportion of nulliparous mothers, who smoked during pregnancy and experienced mental health problems during pregnancy compared to children not in the borderline/clinical range (Table S3).

Children prenatally exposed to acetaminophen were overall more likely to have older and non-nulliparous mothers with higher education levels and higher pre-pregnancy BMI who report alcohol consumption, smoking and mental health problems during pregnancy (Table S4). Children postnatally exposed to acetaminophen were more likely to have nulliparous mothers, who have higher education levels and report alcohol consumptions during pregnancy (Table S5).

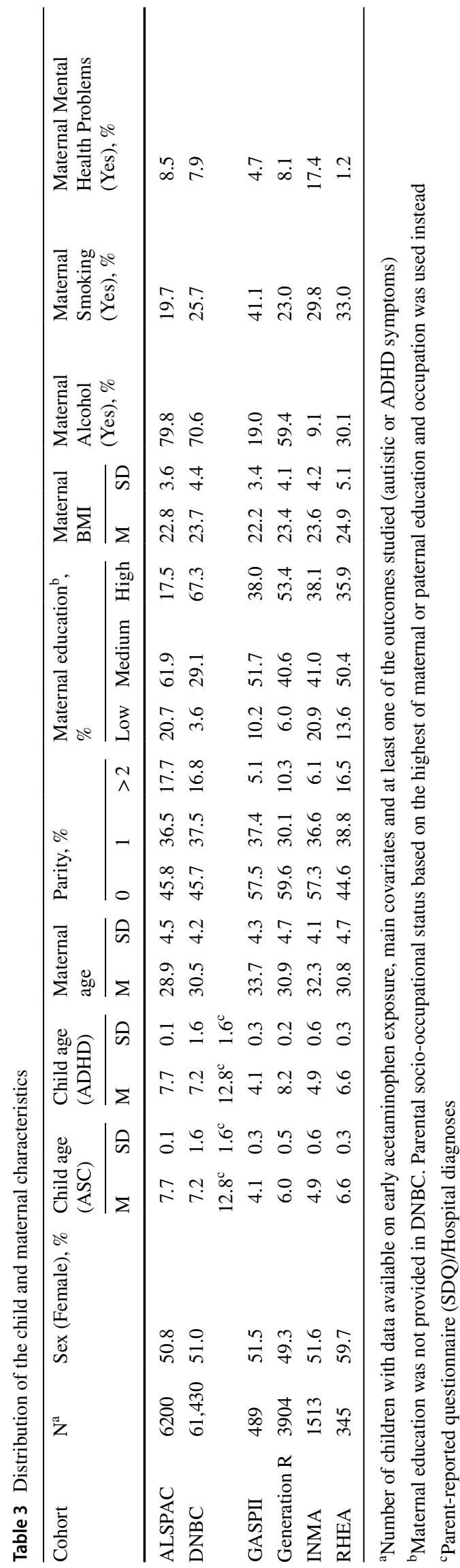




\section{Prenatal acetaminophen exposure and ASC symptoms}

Children prenatally exposed to acetaminophen were 19\% more likely to subsequently have ASC symptoms within the borderline/clinical range than non-exposed children (OR $=1.19,95 \%$ CI 1.07-1.33) (Fig. 1). Similar results were observed using hospital diagnosis in DNBC cohort $(\mathrm{OR}=1.16,95 \%$ CI 1.05-1.29) (Fig. S1). When stratifying by sex, prenatal acetaminophen was associated with ASC symptoms among boys $(\mathrm{OR}=1.28,95 \% \mathrm{CI} 1.12,1.46)$ and to a lesser extent among girls $(\mathrm{OR}=1.06,95 \%$ CI 0.82 , 1.36) (Table 4), though there was no statistical evidence of a difference between boys and girls $\left(P_{\text {interaction }}=0.188\right)$. When using hospital diagnosis in DNBC, a similar effect size was observed in boys $(\mathrm{OR}=1.14,95 \%$ CI $1.00,1.29)$ and girls $(\mathrm{OR}=1.15,95 \%$ CI $0.78,1.71)$ (Table S6). Excluding ADHD cases, prenatal acetaminophen exposure was associated with ASC symptoms based on questionnaires in all cohorts $(\mathrm{OR}=1.16,95 \%$ CI 1.01, 1.43) (Table S7). Additional adjustment for other confounders did not change the results meaningfully (Table S8). In leave-one-out analyses results, the association was attenuated but remained positive when omitting DNBC $(\mathrm{OR}=1.12,95 \%$ CI $0.96,1.30$ while no changes were observed when removing each of the other cohorts (Table S9).

Substantial between-study heterogeneity was only observed when examining this association among girls using hospital diagnosis in DNBC $\left(I^{2}=68.37 \%\right)$ (Table S6).

Meta-regression analyses showed that children's age at outcome assessment did not modified the association between prenatal exposure to acetaminophen and ASC symptoms $(P$-value $=0.923)$.

\section{Prenatal acetaminophen exposure and ADHD symptoms}

The odds of developing ADHD symptoms within the borderline/clinical range were $21 \%$ higher among children prenatally exposed to acetaminophen compared to non-exposed children $(\mathrm{OR}=1.21,95 \%$ CI 1.07-1.36) (Fig. 1). Prenatal acetaminophen use was associated with ADHD symptoms in boys $(\mathrm{OR}=1.23,95 \% \mathrm{CI} 1.05,1.44)$ and to a similar extent in girls $(\mathrm{OR}=1.18,95 \% \mathrm{CI} 0.97,1.44)\left(P_{\text {interaction }}=0.747\right)$ (Table 4). In sensitivity analysis, we observed positive associations using hospital diagnoses in DNBC (OR $=1.30$, 95\% CI 1.14, 1.48) (Fig. S1) and consistent results in boys $(\mathrm{OR}=1.31,95 \% \mathrm{CI} 1.15,1.49)$ and girls $(\mathrm{OR}=1.39,95 \%$ CI $1.15,1.68$ ) (Table S6). Results were also similar with
(A) Prenatal acetaminophen exposure and ASD symptoms

\begin{tabular}{|c|c|c|c|c|c|c|c|}
\hline Cohort & $\mathbf{N}$ & Cases & Exposed & Cases Exposed & & Weight & OR $[95 \% \mathrm{CI}]$ \\
\hline ALSPAC & 5867 & 396 & 2325 & 150 & + & $24.96 \%$ & $1.08[0.87,1.34]$ \\
\hline DNBC & 61430 & 729 & 34584 & 464 & $=$ & $47.41 \%$ & $1.28[1.10,1.49]$ \\
\hline GASP & 489 & 63 & 153 & 10 & - & $\mathbf{3 . 0 7} \%$ & $1.04[0.55,1.95]$ \\
\hline GenerationR & 3689 & 293 & 1098 & 98 & $=$ & $17.29 \%$ & $1.25[0.96,1.62]$ \\
\hline INMA & 1513 & 99 & 775 & 48 & 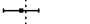 & $6.04 \%$ & $0.89[0.57,1.39]$ \\
\hline RHEA & 308 & 31 & 45 & 6 & 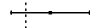 & $1.23 \%$ & $1.87[0.69,5.08]$ \\
\hline \multicolumn{5}{|c|}{$\begin{array}{l}\text { Overall Random Effects Model } \\
\left.\text { RE Model for All Studies (Q=4.34, df }=5, p=0.50 ; I^{2}=2.4 \%\right) ; \tau^{2}=0.00\end{array}$} & & $100.00 \%$ & $1.19[1.07,1.33]$ \\
\hline & & & & $\Gamma$ & 111 & & \\
\hline & & & & 0.05 & $\begin{array}{lll}1 & 2 & 4\end{array}$ & & \\
\hline
\end{tabular}

(C) Postnatal acetaminophen exposure and ASD symptoms

\begin{tabular}{|c|c|c|c|c|c|c|c|}
\hline Cohort & $\mathbf{N}$ & Cases & Exposed & d Cases Exposed & & Weight & OR $[95 \% \mathrm{CI}]$ \\
\hline ALSPAC & 5868 & 389 & 358 & 30 & $\rightarrow$ & $24.20 \%$ & $0.81[0.54,1.20]$ \\
\hline DNBC & 48161 & 585 & 4710 & 76 & in & $31.84 \%$ & $1.30[1.02,1.66]$ \\
\hline GASP & 488 & 63 & 453 & 57 & & $7.41 \%$ & $1.10[0.39,3.06]$ \\
\hline GenerationR & 2267 & 159 & 1941 & 142 & $\stackrel{-}{\vdots}$ & $18.23 \%$ & $1.38[0.81,2.36]$ \\
\hline INMA & 979 & 61 & 748 & 41 & $=$ & $16.01 \%$ & $0.68[0.37,1.23]$ \\
\hline RHEA & 243 & 20 & 237 & 18 & 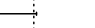 & $2.31 \%$ & $0.15[0.02,1.07]$ \\
\hline \multicolumn{5}{|c|}{$\begin{array}{l}\text { Overall Random Effects Model } \\
\text { RE Model for All Studies }\left(\mathrm{Q}=11.34, \mathrm{df}=5, \mathrm{p}=0.05 ; \mathrm{I}^{2}=48.4 \%\right) ; \mathrm{t}^{2}=0.06\end{array}$} & & $100.00 \%$ & $0.99[0.73,1.35]$ \\
\hline & & & & & $\begin{array}{lll}1 & 1 \\
1 \quad 24\end{array}$ & & \\
\hline
\end{tabular}

Fig. 1 Associations between early acetaminophen exposure and autistic autism spectrum condition (ASC) (a, c) and attention-deficit and hyperactivity (ADHD) symptoms (b, d) within the borderline/clinical range. Associations for prenatal $(\mathbf{a}, \mathbf{b})$ and postnatal $(\mathbf{c}, \mathbf{d})$ exposure are shown. Symptoms were assessed using parent and teacher reported questionnaires in all cohorts. Odds Ratio (OR) and 95\% confidence intervals (CI) by cohort and overall estimate obtained from random-effects meta-analysis. Models were adjusted for mater-
(B) Prenatal acetaminophen exposure and ADHD symptoms

\begin{tabular}{|c|c|c|c|c|c|c|c|}
\hline Cohort & $\mathbf{N}$ & Cases & Exposed & Cases Exposed & & Weight & OR $[95 \% \mathrm{CI}]$ \\
\hline ALSPAC & 5841 & 116 & 2315 & 43 & 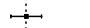 & $9.95 \%$ & $1.01[0.68,1.49]$ \\
\hline DNBC & 61430 & 573 & 34584 & 354 & 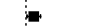 & $51.27 \%$ & $1.22[1.03,1.45]$ \\
\hline GASP & 489 & 50 & 152 & 20 & $\rightarrow$ & $3.55 \%$ & $1.61[0.84,3.10]$ \\
\hline GenerationR & 2864 & 295 & 889 & 110 & $=$ & $22.65 \%$ & $1.25[0.96,1.62]$ \\
\hline INMA & 1239 & 175 & 641 & 97 & 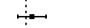 & $11.72 \%$ & $1.16[0.81,1.66]$ \\
\hline RHEA & 317 & 20 & 48 & 3 & $\vdots$ & $0.86 \%$ & $1.11[0.29,4.18]$ \\
\hline \multicolumn{5}{|c|}{$\begin{array}{l}\text { Overall Random Effects Model } \\
\text { RE Model for All Studies }\left(Q=1.72, d f=5, p=0.89: I^{2}=0.0 \%\right): \tau^{2}=0.00\end{array}$} & $\vdots$ & $100.00 \%$ & $1.21[1.07,1.36]$ \\
\hline & & & & & 111 & & \\
\hline & & & & & & & \\
\hline & & & & OR & $\% \mathrm{Cl}]$ & & \\
\hline
\end{tabular}

(D) Postnatal acetaminophen exposure and ADHD symptoms

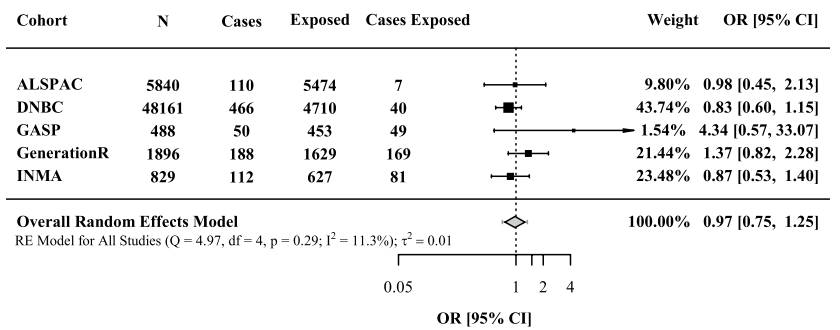

nal characteristics (education, age at delivery, pre-pregnancy body mass index, prenatal smoking, mental health during pregnancy, parity and alcohol consumption, fever and infections during pregnancy) and child's characteristics (sex, age at the behavioural assessment). Postnatal models were further adjusted by child's cold or respiratory infections. Models on postnatal exposure and ADHD symptoms were not possible to conduct in RHEA cohort (limited sample size) 
additional adjustment for confounders (Table S8) or in leaveone-out analysis (Table S9). There was no strong evidence of between-study heterogeneity (Fig. 1, Table 4, Fig. S2, Tables S6-S9).

Meta-regression analyses showed that children's age at outcome assessment did not modified the association between prenatal exposure to acetaminophen and ADHD symptoms $(P$-value $=0.531)$.

\section{Postnatal acetaminophen exposure and ASC symptoms}

No association was found between postnatal acetaminophen exposure and ASC $(\mathrm{OR}=0.99,95 \%$ CI 0.73-1.35) (Fig. 1). Stratified results by sex were also close to the null for both boys and girls (Table 4, Table S4). Similar results were observed when using hospital diagnosis in DNBC (Fig. 1S). Results were also close to the null when excluding ADHD cases (Table S5) and with additional adjustment for other confounders (Table S6). There was evidence of betweenstudy study heterogeneity in main results $\left(I^{2}=48.4\right)$ (Fig. 1).

Meta-regression analyses showed that children's age at outcome assessment did not modified the association between postnatal exposure to acetaminophen and ASC symptoms $(P$-value $=0.975)$.

\section{Postnatal acetaminophen exposure and ADHD symptoms}

No association was found between postnatal acetaminophen exposure and ADHD symptoms $(\mathrm{OR}=0.97,95 \%$ CI 0.75-1.25) (Fig. 1). Associations were also null when stratifying by sex (Table 4, Table S6). Similar results were observed in sensitivity analysis using hospital diagnosis in DNBC (Fig. S1), with additional adjustment for other confounders (Table S8) and in leave-one-out analysis (Table S10). There was no strong evidence of marked between-study heterogeneity (Fig. 1, Fig S1, Table 4, Tables S6-S10).

Meta-regression analyses showed that children's age at outcome assessment did not modified the association between postnatal exposure to acetaminophen and ADHD symptoms $(P$-value $=0.765)$.

\section{Discussion}

The results of our meta-analysis representing more than 70,000 children of six European population-based birth/ child cohorts indicated that children prenatally exposed to acetaminophen were $19 \%$ and $21 \%$ more likely to subsequently have ASC and ADHD symptoms within the borderline/clinical range, respectively, compared with non-exposed 
children. The association with ASC was attenuated after omitting the largest cohort but remained positive. When stratifying by sex, these associations were slightly stronger among boys compared to girls but positive associations with effect sizes of similar magnitude were observed in both strata, especially in the case of ADHD. Postnatal exposure to acetaminophen was not associated with either of the outcome, thought there was evidence of between-study heterogeneity for the association with ASC symptoms.

The most consistent pattern of results was observed for the association between prenatal acetaminophen exposure and ADHD symptoms. The positive associations were observed in all the cohorts and of similar magnitude regardless of the cohort excluded in the leave-one-out analysis. This finding is in agreement with previous meta-analysis which reported likelihood increases of $25 \%$ and $34 \%$ for ADHD in relation to prenatal acetaminophen exposure $[12,13]$. Our findings are consistent with previous single cohort studies conducted in ALSPAC [7], DNBC [1,9] and INMA [6] cohorts, which were included in our meta-analysis. Despite the overlap of samples included, this agreement supports the robustness of the findings since analytical strategies and outcome definitions were harmonized for the present meta-analysis.

The association between prenatal acetaminophen use and ASC symptoms was consistently positive even after omitting the largest cohort. Previous findings in DNBC only found this association in ASC cases with hyperactive symptoms [9], however, in our meta-analysis the association remained after excluding ADHD cases. Overall our findings provide support for the association between prenatal acetaminophen and ASC symptoms in line with a previous meta-analysis [12].

Associations between prenatal acetaminophen and ASC and ADHD symptoms were consistently positive for both boys and girls albeit slightly stronger among boys, with near identical odds ratios for hospital diagnosed cases in DNBC. Importantly, we found no evidence for statistical interaction between child sex and prenatal acetaminophen exposure and either ASC or ADHD symptoms. This contrasts with previous findings in single cohort studies reporting sex differences in the association between prenatal acetaminophen and ASC $[6,9,16]$ and ADHD $[8,16]$. Our findings suggest that differential sex effects of acetaminophen on ASC and ADHD symptoms, if any, are modest and may be dependent on the number of cases, outcome definition and assessment.

Associations between postnatal acetaminophen exposure and both ASC and ADHD symptoms were close to the null and different directions of associations around the null were observed across cohorts. Heterogeneity was high in the case of ASC, with about $50 \%$ of the variation in odds of ASC symptoms being explained by between study differences. The high prevalence of the exposure to acetaminophen when combining pre- and post-natal across studies made it impossible to explore cumulative effects combining prenatal and postnatal exposures. Previous studies examining postnatal acetaminophen exposure have focused on ASC and results are mixed [17, 32, 33]. We do not find evidence supporting this association in either ASC or ADHD symptoms but further research in larger samples is required.

The mechanisms proposed to underlie the adverse effects of early acetaminophen exposure on neurodevelopment include the stimulation of the endocannabinoid system, changes in brain-derived neurotrophic factor (BDNF) levels, oxidative stress due to inflammation-induced immune activation, changes in neurotransmission and endocrinedisruptive properties of acetaminophen [34, 35]. Acetaminophen exposure during periods equivalent to third trimester of pregnancy in humans but not later, induced behavioural and cognitive alterations in both male and female mice [36]. Other animal studies report findings that may be particularly interesting for ADHD. For instance, maternal exposure to acetaminophen was associated with lower levels of BDNF at the level of the striatum in an animal study conducted in male rats [37]. Furthermore, in male mice, acetaminophen treatment induced alterations in spatial learning, memory and dopamine metabolism [38]. Both the striatum region and dopamine are thought to play a pivotal role in ADHD [39-41].

The abovementioned findings provide biological plausibility and coherence for the current findings. In this regard, other causal criteria supported by the current findings include consistency and temporality [42]. Consistency is supported because we observed consistent results using a variety of populations and methods. Temporality is supported because the exposure precedes the onset of the symptoms assessed. Although we did not address dose-response relationship, previous studies have shown dose-response effects for both ASC and ADHD symptoms. [1, 6, 9]

Our findings need to be interpreted with caution given the limitations of our study. First, ASC and ADHD symptoms were assessed by different instruments in the cohorts. All instruments have been validated for the assessment of these symptoms, but the coverage may slightly differ among the instruments. To overcome this heterogeneity, we used instrument specific cut-offs to evaluate the presence or absence of borderline/clinical symptoms, a strategy that other meta-analysis analysing these outcomes have used [43, 44]. Although cohorts differed in the prevalence of ASC and ADHD symptoms, associations were largely consistent. Of note, despite all instruments used herein are widely used in the field but it would be important to examine the psychometric properties of the instruments used in each cohort to establish the specific objectivity of the outcomes. Second, confounding by indication cannot be completely ruled out although potential indications for acetaminophen use were 
included as covariates (maternal fever or infections during pregnancy, maternal chronic illnesses, and child cold or infections in the first 18 months of life). Third, dose and frequency of use were not harmonized across cohorts and therefore, not analysed herein. Fourth, although results were adjusted by several lifestyles and health factors that have been shown to be associated with prenatal acetaminophen exposure [45], residual confounding by social class cannot be completed discarded. However, the consistent associations found across different sensitivity analysis including examining ASC and ADHD diagnosis in the largest cohort makes unlikely that the observed relationship between prenatal acetaminophen and ASC and ADHD symptoms is entirely explained by unmeasured confounding. Fifth, given the high rates of loss to follow-up in some of the included cohorts, we cannot discard selection bias in some of the cohorts. Nevertheless, loss to follow-up was low $(<2 \%)$ in the largest cohort included when using hospital diagnosis, which had broadly consistent findings compared to all the other cohorts together. Sixth, we have exposure information on postnatal acetaminophen use up to different ages in different cohorts and one regional cohort only collected medication in case of infection, which may have caused some exposure misclassification in that cohort. Finally, although information was prospectively collected, we cannot rule out information bias or misclassification of exposure.

Overall, despite the above limitations, the homogeneity of the findings among the different cohorts, the novel assessment of postnatal acetaminophen exposure, and the use of an harmonized definition of exposure and outcome as well as of common statistical approaches overcomes the criticisms of previous meta-analysis [14, 15].

To conclude, our results support previous findings and address part of the weaknesses of previous meta-analyses. Considering all evidences on acetaminophen use and neurodevelopment, we agree with previous recommendations indicating that while acetaminophen should not be suppressed in pregnant women or children, it should be used only when necessary [46].

Supplementary Information The online version contains supplementary material available at https://doi.org/10.1007/s10654-021-00754-4.

Acknowledgements We are grateful to Dr. Ewen Harrison for his kind help with finalfit package. We are extremely grateful to all of the families who took part in ALSPAC, the midwives for their help in recruiting them, and the whole ALSPAC team, which includes interviewers, computer and laboratory technicians, clerical workers, research scientists, volunteers, managers, receptionists and nurses. We are grateful to all the participating families in DNBC and the general practitioners and midwifes for enrolling the pregnant women back in time. Further, our small and effective steady study secretariat deserves acknowledgment. The Generation R Study is conducted by the Erasmus Medical Centre in close collaboration with the Municipal Health Service Rotterdam area, the Rotterdam Homecare Foundation and the Stichting Trombosedienst \& Artsenlaboratorium Rijnmond (STAR), Rotterdam.
We gratefully acknowledge the contribution of general practitioners, hospitals, midwives and pharmacies in Rotterdam. GASPII, INMA and RHEA cohorts thank all participants for their generous collaboration.

Author contributions SA conceptualized and designed the study, conducted analysis, interpretated the data, drafted the initial manuscript, and reviewed and revised the manuscript. CA and ZL made substantial contributions to the conceptualization and design of the study and the initial draft and critically reviewed the manuscript for important intellectual content. RGE, KI, TC, MLV conducted analysis, interpreted the data and reviewed and revised the manuscript. LG, IRG, AA, MC, KM and $\mathrm{JJ}$ contributed to the design of the data collection instruments, data collection, interpreted the data and critically reviewed the manuscript for important intellectual content. KSL, DAL, HEM, HT, CI, AT, LSM, DP and LC coordinated and supervised data collection, acquire funding and critically reviewed the manuscript for important intellectual content. JS conceptualized and designed the study, critically reviewed the manuscript for important intellectual content and provided final approval of the version to be published.

Funding The Avon Longitudinal Study of Parents and Children (ALSPAC) receives core funding from the University of Bristol, UK Medical Research Council and Wellcome (217065/Z/19/Z). A comprehensive list of grants funding is available on the ALSPAC website (http://www.bristol.ac.uk/alspac/external/documents/grant-ackno wledgements.pdf). DAL's contribution to this paper is supported by the European Research Council under the European Union's Seventh Framework Programme (FP7/2007-2013/ERC grant agreement no 669545) and a UK National Institute of Health Senior Investigator (NF-0616-10102). TC's contribution is supported by European Union's Horizon 2020 research and innovation programme under grant agreement No 733206 (LifeCycle). DAL and TC work in a Unit that is supported by the University of Bristol and UK Medical Research Council (MC_UU_00011/6). The Danish National Birth Cohort (DNBC) was established with a significant grant from the Danish National Research Foundation. Additional support was obtained from the Danish Regional Committees, the Pharmacy Foundation, the Egmont Foundation, the March of Dimes Birth Defects Foundation, the Health Foundation and other minor grants. The DNBC Biobank has been supported by the Novo Nordisk Foundation and the Lundbeck Foundation. Follow-up of mothers and children have been supported by the Danish Medical Research Council (SSVF 0646, 27108-0839/06-066023, O602-01042B, 0602-02738B), the Lundbeck Foundation (195/04, R100-A9193), the Innovation Fund Denmark 0603-00294B (09-067124), the Nordea Foundation (02-2013-2014), Aarhus Ideas (AU R9-A959-13-S804), University of Copenhagen Strategic Grant (IFSV 2012), and the Danish Council for Independent Research (DFF-4183-00594 and DFF-4183-00152). The Gene and Environment: Prospective Study on Infancy in Italy (GASPII) was funded by the Italian Ministry of Health and by the Italian Medicines Agency. The general design of the Generation R Study is made possible by financial support from the Erasmus Medical Center, Rotterdam, the Erasmus University Rotterdam, the Netherlands Organization for Health Research and Development (ZonMw), the Netherlands Organization for Scientific Research (NWO), the Ministry of Health, Welfare, and Sport, and the Ministry of Youth and Families. This study was supported by the NARSAD Young Investigator Grant from the Brain $\&$ Behavior Research Foundation grant number 27853 (HEM), Vici project 016.VICI.170.200 (HT). MLV has received funding from the European Union's Horizon 2020 research and innovation programme under the Marie Skłodowska-Curie grant agreement No 707404. The opinions expressed in this document reflect only the author's view. The European Commission is not responsible for any use that may be made of the information it contains. The INfancia y Medio Ambiente (INMA)-Sabadell cohort was funded by grants from Instituto de 
Salud Carlos III (Red INMA G03/176; CB06/02/0041; CP18/00018; PI041436; PI081151; PI1100610 incl. FEDER funds), Generalitat de Catalunya-CIRIT 1999SGR 00241, Fundació La marató de TV3 (090430). ISGlobal acknowledge support from the Spanish Ministry of Science and Innovation through the "Centro de Excelencia Severo Ochoa 2019-2023" Program (CEX2018-000806-S), and support from the Generalitat de Catalunya through the CERCA Program. SA is funded by a Juan de la Cierva-Incorporación Postdoctoral Contract awarded by Ministry of Economy, Industry and Competitiveness (IJCI-2017-34068). JJ holds Miguel Servet-II contract (CPII19/00015) awarded by the Instituto de Salud Carlos III (Co-funded by European Social Fund "Investing in your future"). MC holds a Miquel ServetI cotract (CP16/00128) awarded by the Instituto de Salud Carlos III (co-funded by European Social Fund "Investing in your future"). The INMA-Asturias cohort is funded by grants from Instituto de Salud Carlos III (FISS PI 04/2018, FIISPI09/02311, FISSPI13/02429, FISS PI18/00909 including FEDER funds) and University of Oviedo. This study was funded by Instituto de Salud Carlos III through the projects 'CP14/00108 \& PI16/00261' (co-funded by European Regional Development Fund 'A way to make Europe') and CIBERESP, Obra Social Cajastur/Fundación Liberbank. The INMA-Gipuzkoa was funded by grants from Instituto de Salud Carlos III (FIS-PI06/0867, FIS-PI09/00090, FIS-PI18/01142 and FIS-PI13/02187 incl. FEDER funds), Department of Health of the Basque Government (2005111093, 2009111069, 2013111089 and 2015111065), and the Provincial Government of Gipuzkoa (DFG06/002, DFG08/001 and DFG15/221) and annual agreements with the municipalities of the study area (Zumarraga, Urretxu, Legazpi, Azkoitia y Azpeitia y Beasain). The INMAValencia was funded by Grants from European Union (FP7-ENV-2011 cod 282957 and HEALTH.2010.2.4.5-1), Instituto de Salud Carlos III (Red INMA G03/176, CB06/02/0041; FIS-FEDER: PI03/1615, PI04/1509, PI04/1112, PI04/1931, PI05/1079, PI05/1052, PI06/1213, PI07/0314, PI09/02647, PI11/01007, PI11/02591, PI11/02038, PI13/1944, PI13/2032, PI14/00891, PI14/01687, PI16/1288, and PI17/00663; Miguel Servet-FEDER CP11/00178, CP15/00025, and CPII16/00051), Generalitat Valenciana: FISABIO (UGP 15-230, UGP15-244, and UGP-15-249), and Alicia Koplowitz Foundation 2017. The Rhea project was financially supported by European projects (EU FP6-2003-Food-3-NewGeneris, EU FP6. STREP Hiwate, EU FP7 ENV.2007.1.2.2.2. Project No 211250 Escape, EU FP7- 2008-ENV1.2.1.4 Envirogenomarkers, EU FP7-HEALTH- 2009- single stage CHICOS, EU FP7 ENV.2008.1.2.1.6. Proposal No 226285 ENRIECO, EUFP7- HEALTH-2012 Proposal No 308333 HELIX, FP7 European Union project, No. 264357 MeDALL), and the Greek Ministry of Health (Program of Prevention of obesity and neurodevelopmental disorders in preschool children, in Heraklion district, Crete, Greece: 2011-2014; Rhea Plus: Primary Prevention Program of Environmental Risk Factors for Reproductive Health, and Child Health: 2012-15). LC was supported by the National Institute of Environmental Health Sciences (R01ES029944, R01ES030364, R21ES28903, R21ES029681, P30ES007048).

Code availability Customized scripts in R software are available from the corresponding author upon request.

Data availability In order to protect participant's privacy, data at individual level cannot be made publicly available. For further details, authors can be contacted at http://www.isglobal.org/ca/.

\section{Declarations}

Conflict of interest DA Lawlor declares that she has received support for research unrelated to this project from numerous national and inter- national charity and government funders and from Medtronic Ltd and Roche Diagnostics. The rest of authors declare no conflict of interest.

Open Access This article is licensed under a Creative Commons Attribution 4.0 International License, which permits use, sharing, adaptation, distribution and reproduction in any medium or format, as long as you give appropriate credit to the original author(s) and the source, provide a link to the Creative Commons licence, and indicate if changes were made. The images or other third party material in this article are included in the article's Creative Commons licence, unless indicated otherwise in a credit line to the material. If material is not included in the article's Creative Commons licence and your intended use is not permitted by statutory regulation or exceeds the permitted use, you will need to obtain permission directly from the copyright holder. To view a copy of this licence, visit http://creativecommons.org/licenses/by/4.0/.

\section{References}

1. Liew Z, Ritz B, Rebordosa C, Lee P-C, Olsen J. Acetaminophen use during pregnancy, behavioral problems, and hyperkinetic disorders. JAMA Pediatr. 2014;168:313-20.

2. Brandlistuen RE, Ystrom E, Nulman I, Koren G, Nordeng H. Prenatal paracetamol exposure and child neurodevelopment: a sibling-controlled cohort study. Int J Epidemiol. 2013;42:1702-13.

3. Thompson JMD, Waldie KE, Wall CR, Murphy R, Mitchell EA, $\mathrm{ABC}$ study group. Associations between acetaminophen use during pregnancy and ADHD symptoms measured at ages 7 and 11 years. PLoS ONE. 2014;9:e108210 (Hashimoto K, editor).

4. Liew Z, Ritz B, Virk J, Arah OA, Olsen J. Prenatal use of acetaminophen and child IQ: A danish cohort study. Epidemiology. 2016;27:912-8.

5. Liew Z, Bach CC, Asarnow RF, Ritz B, Olsen J. Paracetamol use during pregnancy and attention and executive function in offspring at age 5 years. Int J Epidemiol. 2016;45:2009-17.

6. Avella-Garcia CB, Julvez J, Fortuny J, Rebordosa C, GarcíaEsteban R, Galán IR, et al. Acetaminophen use in pregnancy and neurodevelopment: attention function and autism spectrum symptoms. Int J Epidemiol. 2016;1-9.

7. Stergiakouli E, Thapar A, Davey SG. Association of acetaminophen use during pregnancy with behavioral problems in childhood: evidence against confounding. JAMA Pediatr. 2016;170:964-70.

8. Tovo-Rodrigues L, Schneider BC, Martins-Silva T, Del-Ponte B, Loret De Mola C, Schuler-Faccini L, et al. Is intrauterine exposure to acetaminophen associated with emotional and hyperactivity problems during childhood? findings from the Pelotas birth cohort. BMC Psychiatry. BioMed Cent. 2018;18:368.

9. Liew Z, Ritz B, Virk J, Olsen J. Maternal use of acetaminophen during pregnancy and risk of autism spectrum disorders in childhood: A Danish national birth cohort study. Autism Res. 2016;9:951-8.

10. Chen M-H, Pan T-L, Wang P-W, Hsu J-W, Huang K-L, Su T-P, et al. Prenatal exposure to acetaminophen and the risk of attentiondeficit/hyperactivity disorder. J Clin Psychiatry. 2019;80:01-3.

11. Baker BH, Lugo-Candelas $\mathrm{C}, \mathrm{Wu} \mathrm{H}$, Laue HE, Boivin A, Gillet $\mathrm{V}$, et al. Association of prenatal acetaminophen exposure measured in meconium with risk of attention-deficit/hyperactivity disorder mediated by frontoparietal network brain connectivity. JAMA Pediatr. 2020;174(11):1073-81.

12. Masarwa R, Levine H, Gorelik E, Reif S, Perlman A, Matok I. Prenatal exposure to acetaminophen and risk for attention deficit hyperactivity disorder and autistic spectrum disorder: a 
systematic review, meta-analysis, and meta-regression analysis of cohort studies. Am J Epidemiol. 2018;187:1817-27.

13. Gou X, Wang Y, Tang Y, Qu Y, Tang J, Shi J, et al. Association of maternal prenatal acetaminophen use with the risk of attention deficit/hyperactivity disorder in offspring: A meta-analysis. Aust New Zeal J Psychiatry. 2019;53(3):195-206.

14. Gilman SE, Hornig M. The disillusionment of DOHaD epidemiology. Am J Epidemiol. 2020;189(1):1-5.

15. Damkier P. RE: prenatal exposure to acetaminophen and risk for attention deficit hyperactivity disorder and autistic spectrum disorder: a systematic review, meta-analysis, and meta-regression analysis of cohort studies. Am J Epidemiol. 2018;187(8):1817-1827.

16. Ji Y, Azuine RE, Zhang Y, Hou W, Hong X, Wang G, et al. Association of cord plasma biomarkers of in utero acetaminophen exposure with risk of attention-deficit/hyperactivity disorder and autism spectrum disorder in childhood. JAMA Psychiat. 2019;77(2):180-9.

17. Bauer AZ, Kriebel D. Prenatal and perinatal analgesic exposure and autism: an ecological link. Environ Heal A Glob Access Sci Source. 2013;12:41.

18. Goodman R, Ford T, Richards H, Gatward R, Meltzer H. The Development and well-being assessment: description and initial validation of an integrated assessment of child and adolescent psychopathology. J Child Psychol Psychiatry. 2000;41:645-55.

19. Achenbach T, Rescorla L. Manual for the ASEBA preschool forms \& profiles. Burlington: University of Vermont, Research Center for Children, Youth, \& Families; 2000.

20. Scott FJ, Baron-Cohen S, Bolton P, Brayne C. The CAST (childhood asperger syndrome test): preliminary development of a UK screen for mainstream primary-school-age children. Autism. 2002;6:9-31.

21. Ooi YP, Rescorla L, Ang RP, Woo B, Fung DSS. Identification of autism spectrum disorders using the child behavior checklist in Singapore. J Autism Dev Disord. 2011;41:1147-56.

22. Conners CK. Conner's rating scales-revised user's manual. New York: Multi-Health Systems, North Tonawanda; 1997.

23. Goodman R. The strengths and difficulties questionnaire: a research note. J Child Psychol Psychiatry Allied Discip. 1997;38:581-6.

24. American Psychiatric Association. Diagnostic and statistical manual of mental disorders (DSM-5). 5th ed. Washington DC: American Psychiatric Association; 2013.

25. Tick NT, Van der Ende J, Koot HM, Verhulst FC. 14-Year changes in emotional and behavioral problems of very young Dutch children. J Am Acad Child Adolesc Psychiatry. 2007;46:1333-40.

26. Achenbach T, Rescorla LA. Manual for the ASEBA schoolage forms \& profiles. Burlington, VT: University of Vermont, Research Center for Children, Youth \& Families; 2001.

27. Foreman D, Morton S, Ford T. Exploring the clinical utility of the development and well-being assessment (DAWBA) in the detection of hyperkinetic disorders and associated diagnoses in clinical practice. J Child Psychol Psychiatry Allied Discip. 2009;50:460-70.

28. Russell G, Rodgers LR, Ford T. The strengths and difficulties questionnaire as a predictor of parent-reported diagnosis of autism spectrum disorder and attention deficit hyperactivity disorder. PLoS ONE. 2013;8:e80247. https://doi.org/10.1371/journal.pone. 0080247 (Senju A, editor).

29. Skuse DH, Mandy W, Steer C, Miller LL, Goodman R, Lawrence $\mathrm{K}$, et al. Social communication competence and functional adaptation in a general population of children: preliminary evidence for sex-by-verbal IQ differential risk. J Am Acad Child Adolesc Psychiatry. 2009;48:128-37.

30. Riley RD, Higgins JPT, Deeks JJ. Interpretation of random effects meta-analyses. BMJ Br Med J Publ Group. 2011;342:964-7.
31. Viechtbauer W. Conducting meta-analyses in $\mathrm{R}$ with the metafor. J Stat Softw. 2010;36:1-48.

32. Schultz ST, Klonoff-Cohen HS, Wingard DL, Akshoomoff NA, Macera CA, Ming J. Acetaminophen (paracetamol) use, measlesmumps-rubella vaccination, and autistic disorder: the results of a parent survey. Autism. 2008;12:293-307.

33. Schultz ST, Gould GG. Acetaminophen use for fever in children associated with autism spectrum disorder. Autism-open Access. 2016;6:170

34. de Fays L, Van Malderen K, De Smet K, Sawchik J, Verlinden V, Hamdani J, et al. Use of paracetamol during pregnancy and child neurological development. Dev Med Child Neurol. 2015;57(8):718-24.

35. Bauer AZ, Kriebel D, Herbert MR, Bornehag CG, Swan SH. Prenatal paracetamol exposure and child neurodevelopment: a review. Horm Behav. 2018;101:125-47.

36. Philippot G, Gordh T, Fredriksson A, Viberg H. Adult neurobehavioral alterations in male and female mice following developmental exposure to paracetamol (acetaminophen): characterization of a critical period. J Appl Toxicol. 2017;37:1174-81.

37. Blecharz-Klin K, Wawer A, Jawna-Zboińska K, Pyrzanowska J, Piechal A, Mirowska-Guzel D, et al. Early paracetamol exposure decreases brain-derived neurotrophic factor (BDNF) in striatum and affects social behaviour and exploration in rats. Pharmacol Biochem Behav. 2018;168:25-32.

38. Blecharz-Klin K, Piechal A, Pyrzanowska J, Joniec-Maciejak I, Kiliszek P, Widy-Tyszkiewicz E. Paracetamol-The outcome on neurotransmission and spatial learning in rats. Behav Brain Res. 2013;253:157-64.

39. Hoogman M, Bralten J, Hibar DP, Mennes M, Zwiers MP, Schweren LSJ, et al. Subcortical brain volume differences in participants with attention deficit hyperactivity disorder in children and adults: a cross-sectional mega-analysis. Lancet Psychiatry. 2017;4:310-9.

40. Liao C, Laporte AD, Spiegelman D, Akçimen F, Joober R, Dion $\mathrm{PA}$, et al. Transcriptome-wide association study of attention deficit hyperactivity disorder identifies associated genes and phenotypes. Nat Commun. 2019;10:1-7.

41. Klein M, Walters RK, Demontis D, Stein JL, Hibar DP, Adams $\mathrm{HH}$, et al. Genetic markers of ADHD-related variations in intracranial volume. Am J Psychiatry. 2019;176:228-38.

42. Hill AB. The environment and disease: association or causation? Proc R Soc Med. 1965;58(5): 295-300.

43. Guxens M, Ghassabian A, Gong T, Garcia-Esteban R, Porta D, Giorgis-Allemand L, et al. Air pollution exposure during pregnancy and childhood autistic traits in four European populationbased cohort studies: the ESCAPE project. Environ Health Perspect. 2016;24(1):133-40.

44. Forns J, Sunyer J, Garcia-Esteban R, Porta D, Ghassabian A, Giorgis-Allemand L, et al. Air pollution exposure during pregnancy and symptoms of attention deficit and hyperactivity disorder in children in europe. Epidemiol NLM (Medline). 2018;29:618-26.

45. Golding J, Gregory S, Clark R, Ellis G, Iles-Caven Y, Northstone $\mathrm{K}$. Associations between paracetamol (acetaminophen) intake between 18 and 32 weeks gestation and neurocognitive outcomes in the child: a longitudinal cohort study. Paediatr Perinat Epidemiol. 2019;34(3):257-66.

46. Toda K. Is acetaminophen safe in pregnancy? Scand J Pain. 2017; 17:445-6.

Publisher's Note Springer Nature remains neutral with regard to jurisdictional claims in published maps and institutional affiliations. 


\section{Authors and Affiliations}

\section{Silvia Alemany 1,2,3 (D) Claudia Avella-García ${ }^{1,2,3,4} \cdot$ Zeyan Liew $^{5,6} \cdot$ Raquel García-Esteban $^{1,2,3} \cdot$ Kosuke Inoue $^{7}$ Tim Cadman ${ }^{8,9}$ • Mònica López-Vicente ${ }^{10}$. Llúcia González ${ }^{3,11}$ • Isolina Riaño Galán ${ }^{3,12}$ • Ainara Andiarena ${ }^{13,14}$. Maribel Casas ${ }^{1,2,3} \cdot$ Katerina Margetaki $^{15} \cdot$ Katrine Strandberg-Larsen $^{16}$. Deborah A. Lawlor ${ }^{8,9,17}$. Hanan El Marroun ${ }^{10,18,19}$. Henning Tiemeier ${ }^{10,20}$. Carmen Iñiguez ${ }^{21,3,11}$. Adonina Tardón ${ }^{3,22}$. Loreto Santa-Marina ${ }^{3,23,24}$. Jordi Júlvez ${ }^{1,2,3,25}$. Daniela Porta ${ }^{26}$. Leda Chatzi ${ }^{27}$. Jordi Sunyer ${ }^{1,2,3,28}$}

1 ISGlobal, Barcelona Institute for Global Health, C. Doctor Aiguader 88, 08003 Barcelona, Spain

2 Universitat Pompeu Fabra (UPF), Barcelona, Spain

3 CIBER Epidemiology and Public Health (CIBERESP), Madrid, Spain

4 Hospital Sagrat Cor, Martorell, Spain

5 Departmen of Environmental Health Sciences, Yale School of Public Health, New Haven, USA

$6 \quad$ Yale Center for Perinatal, Pediatric, and Environmental Epidemiology, Yale School of Public Health, New Haven, USA

7 Department of Epidemiology, Fielding School of Public Health, University of California, Los Angeles (UCLA), Los Angeles, USA

$8 \quad$ MRC Integrative Epidemiology Unit (IEU) and School of Social and Community Medicine, University of Bristol, Bristol, UK

9 Bristol Medical School, Population Health Science, Bristol, UK

10 Department of Child and Adolescent Psychiatry, Erasmus MC-Sophia, Rotterdam, The Netherlands

11 Epidemiology and Environmental Health Joint Research Unit, FISABIO-Universitat Jaume I-Universitat de València, Valencia, Spain

12 Paediatrics, Hospital Universitario Central de Asturias, University of Oviedo and ISPA, Oviedo, Spain

13 Faculty of Psychology, University of the Basque Country, Gipuzkoa, Spain

14 Health Research Institute, Biodonostia, San Sebastian, Spain
15 Department of Social Medicine, University of Crete, Crete, Greece

16 Section for Epidemiology, Department of Public Health, University of Copenhagen, Copenhagen, Denmark

17 Bristol NIHR Biomedical Research Centre, Bristol, UK

18 Department of Pediatrics, University Medical Center Rotterdam, Erasmus MC, Rotterdam, The Netherlands

19 Department of Psychology, Education and Child Studies, Erasmus School of Social and Behavioral Sciences, Erasmus University Rotterdam, Rotterdam, The Netherlands

20 Department of Social and Behavioral Science, Harvard TH Chan School of Public Health, Boston, USA

21 Department of Statistics and Computational Research, Universitat de València, València, Spain

22 Health Research Institute of the Principality of Asturias (ISPA), IUOPA, University of Oviedo, Oviedo, Spain

23 Health Research Institute, Biodonostia, San Sebastian, Spain

24 Public Health Division of Gipuzkoa, Basque Government, Gipuzkoa, Spain

25 Institut d'Investigació Sanitària Pere Virgili (IISPV), Hospital Universitari Sant Joan de Reus, Reus, Catalonia, Spain

26 Department of Epidemiology, Lazio Regional Health Service, Rome, Italy

27 Department of Preventive Medicine, University of Southern California, University Park Campus, Los Angeles, USA

28 IMIM (Hospital del Mar Medical Research Institute), Barcelona, Spain 